\title{
Two Cases of Aquagenic Wrinkling of the Palms in Korean Healthy Women: Easily Overlooked Disease
}

\author{
Hoon Choi, In Ho Bae, Min Sung Kim, Chan Ho Na, Bong Seok Shin \\ Department of Dermatology, Chosun University Medical School, Gwangju, Korea
}

Aquagenic wrinkling of the palms (AWP) is a rare condition, which is characterized by appearance of whitish papules and plaques and an excessive wrinkling and swelling of the palmar skin after exposure to water. Herein, we report two cases of AWP. A 17-year-old woman presented prickling focal various sized whitish papules, plaques with wrinkles on both palms after water contact for 6 months ago. Histologic findings were consistent with AWP. A 26-year-old woman presented asymptomatic multiple various sized whitish papules, plaques with wrinkles on both palms after water contact for 1 year ago. Both patients underwent a cystic fibrosis transmembrane conduct receptor test but were negative and improved without any treatment. (Ann Dermatol 32(2) 151 154, 2020)

\section{-Keywords-}

Aquagenic wrinkling of the palms, Cystic fibrosis

\section{INTRODUCTION}

Aquagenic wrinkling of the palms (AWP) is a rare condition which is characterized by appearance of whitish pap-

Received September 11, 2018, Revised December 10, 2018, Accepted for publication January 2, 2019

Corresponding author: Bong Seok Shin, Department of Dermatology, Chosun University Medical School, 365 Pilmun-daero, Dong-gu, Gwangju 61453, Korea. Tel: 82-62-220-3130, Fax: 82-62-222-3215, E-mail: inho-yoga@hanmail.net

ORCID: https://orcid.org/0000-0001-9618-1763

This is an Open Access article distributed under the terms of the Creative Commons Attribution Non-Commercial License (http://creativecommons. org/licenses/by-nc/4.0) which permits unrestricted non-commercial use, distribution, and reproduction in any medium, provided the original work is properly cited.

Copyright (c) The Korean Dermatological Association and The Korean Society for Investigative Dermatology ules, plaques, an excessive wrinkling, and swelling of the palmar skin after exposure to water about 3 to 5 minutes ${ }^{1}$. In 1964, it was first described by Elliot in cystic fibrosis patient ${ }^{1}$. It is also called Aquagenic acrokertoderma, transient aquagenic palmar hyperwrinkling and aquagenic syringeal acrokeratoderma. It is an easily overlooked if clinician only listen to the patient's history without water contact test and biopsy. In most cases, young women are affected and an association of AWP with cystic fibrosis has been surmised ${ }^{2}$.

\section{CASE REPORT}

\section{Case 1}

A 17-year-old woman presented prickling focal various sized whitish papules, plaques with wrinkles on both palms after water contact for 6 months ago. On the physical examination, whitish discoloration and edematous wrinkling appeared on her palms within 5 minutes water immersion and returned to normal within 30 minutes after dry (Fig. 1A). She had no relevant family or medical history of systemic diseases except localized hyperhidrosis. Histopathologic findings showed hyperkeratosis, dilated eccrine duct with acrosyringia otherwise it seems like normal skin (Fig. 2A). There were no respiratory symptoms, and chest X-ray was normal. She had cystic fibrosis transmembrane conduct receptor (CFTR) test but the test result was negative. She improved without any treatment and is under observation. We received the patient's consent form about publishing all photographic materials.

\section{Case 2}

A 26-year-old woman presented asymptomatic multiple various sized whitish papules, plaques with wrinkles on both palms after water contact for 1 year ago. On the physical examination, whitish discoloration and edematous 
wrinkling appeared on her palms within 5 minutes water immersion (Fig. 1B). She had no relevant family or medical history of systemic disease and chest X-ray was normal. Histopathologic findings showed orthokeratosis, hyperkeratosis and acrosyringia (Fig. 2B). She was negative in the CFTR test and improved without any treatment.

\section{DISCUSSION}

AWP is also known as aquagenic palmoplantar keratoderma, aquagenic syringeal acrokeratoderma, transient reactive papulo-translucent keratoderma or aquagenic acrokeratoderma ${ }^{3}$. AWP mainly occurs in Caucasian adolescent women. The occurrence of AWP in Asian is very rare and up to date all five Asian AWP cases occurred only in young men and screening for CFTR mutations were not evaluated $^{4}$. In these cases, CFTR mutations test was evaluated and all patients were young healthy women. As in
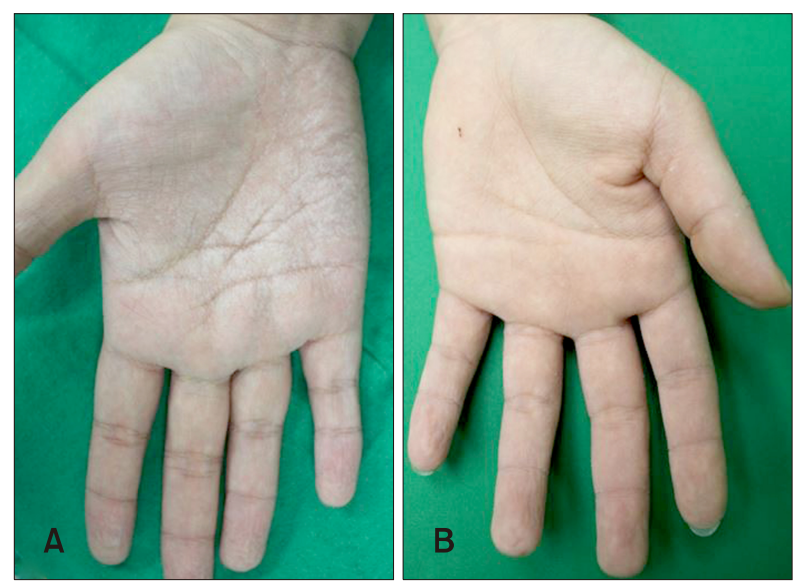

Fig. 1. Clinical images of the palms after the water provocation test. (A) Whitish papules and edematous wrinkling were observed after 5 minutes on a 17-year-old women. (B) Whitish discoloration and edematous wrinkling were observed within 5 minutes on a 26-year-old women. previous Asian cases, our patients were no relation between cystic fibrosis (CF) and drug too. It is often associated with pruritus and a burning or tingling sensation or can be asymptomatic ${ }^{3}$. The diagnosis of AWP is based on clinical findings, which are the rapid development of transient whitish papules and edematous plaques with excessive wrinkling on the palm after exposure to water ("hand-in-the-bucket" sign) ${ }^{5}$. Rarely, they can be seen involving the feet, but the palms are the predominant site of involvement $^{5}$. Palmar wrinkling occurs within 3 minutes after water exposure in AWP whereas water wrinkling is a normal physiologic response to prolonged water contact and occurs at an average 11.5 minutes after water exposure ${ }^{5}$. It can be confused with the Raynaud phenomenon, but it can be easily distinguished by its excessive wrinkling and the irrelevance of cold temperature ${ }^{6,7}$.

Histopathologic examination revealed compact orthokeratosis, hyperkeratosis, acanthosis and dilatation of intracorneal eccrine sweat ducts (acrosyringia) ${ }^{6}$.

Although the precise mechanism is unclear, AWP is assumed to be due to sweat electrolyte disturbances that cause sodium retention in epidermal keratinocytes, leading to an increase in osmotic- induced cell volume ${ }^{3}$. Structural changes in the stratum corneum of AWP result in a malfunction following water contact and an aberrant expression of aquaporin 5 (AQP5) was shown in the sweat glands of AWP patients ${ }^{3}$. Aquaporinis membrane protein that is responsible for rapid osmotic water movement across the plasma membrane. AQP5 may play some roles in sweat secretion ${ }^{3}$. Decreasing desmoglein- 1 and increasing filaggrin in the stratum corneum result in decreased skin barrier function and increased moisturizing factor in AWP. Therefore, Okuhira et al. ${ }^{8}$ suggested that decreasing skin barrier function and water retention by increasing moisturizing factor cause the symptoms of AWP.

Except in Asian cases, most cases AWP was associated with cystic fibrosis. Cystic fibrosis is an autosomal recessive disease caused by mutations in the CFTR gene that
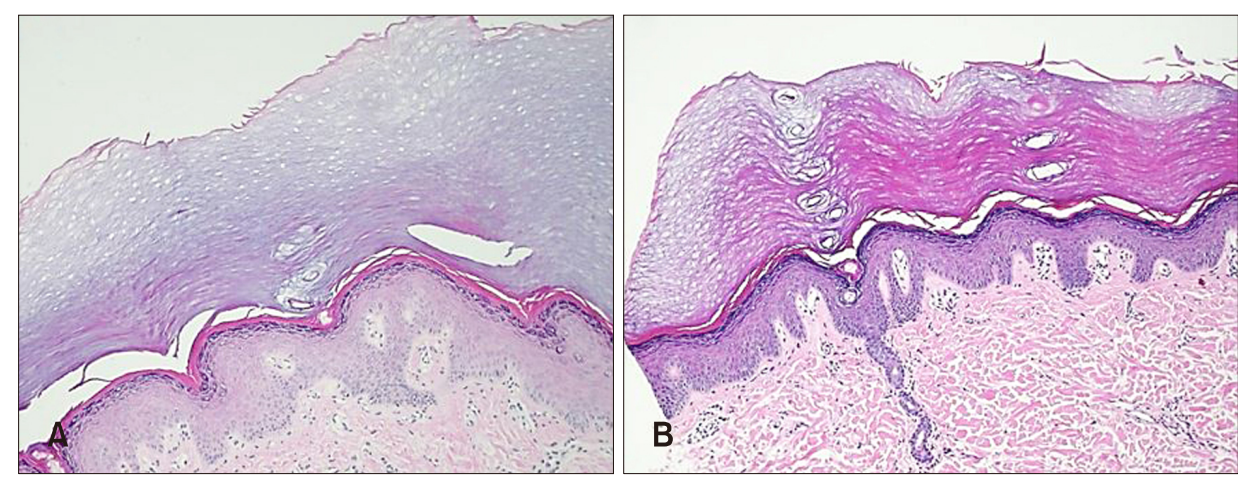

Fig. 2. Histopathological findings of palms. (A) Hyperkeratosis, dilated eccrine duct with acrosyringia after water exposure. (B) Orthokeratosis, hyperkeratosis and acrosyringia after water exposure. (A, B: $\mathrm{H} \& \mathrm{E}$, magnification, $\times 100$ ). 
encodes proteins involved in trans-epithelial ion transport. CF can be found in all ethnic groups and races, however, most often seen in people who are white. AWP presents in up to $84 \%$ of cystic fibrosis patients (symptom develops within $2 \sim 3$ minutes of immersing the hands in water) and $25 \%$ of carriers (symptom typically takes $5 \sim 7$ minutes to appear $)^{9}$. This suggests that AWP is associated with CFTR dysfunction $^{10,11}$. Gild et al. ${ }^{5}$ suggested that patients with AWP should be offered a screening test for both CF and the carrier state due to AWP can be recognition of CF status. In patients with $\mathrm{CF}$, it has been suggested that higher levels of epidermal sodium content may increase the absorption of water above levels of normal persons ${ }^{10,11}$. To date, nearly 2,000 mutations have been identified and represent a variety of CF clinical symptoms depending on the combination. Approximately $5 \%$ of patients are nonclassic CF and have mild clinical symptoms, unlike classic CF. Non-classic CF affects few organs and typically does not present until adolescence or adulthood. As a result, many cases were hard to recognize or diagnosed late ${ }^{12}$. Therefore clinical findings are needed to distinguish nonclassic CF patients, and clinicians should take note of this because AWP can be an early symptom of CF. Not only cystic fibrosis, AWP has been associated with the intake of drug (cyclooxygenase-2 inhibitor, tobramycin) and hyperhidrosis $^{12,13}$. Case 1 patient had a history of localized hyperhidrosis and improved after topical 20\% aluminum chloride hexahydrate for 1 month. AWP patients may benefit from topical application of $15 \% \sim 20 \%$ aluminum chloride solution, iontophoresis and local injection of botulinum toxin ${ }^{6,7}$. Most patients with mild AWP do not require therapy and the condition improves over time during adolescence or young adulthood ${ }^{14}$. In our cases, both patients improved over time without any special treatment. Discontinue any drugs contribute to sweat electrolyte imbalances and systemic acitretin therapy has shown good response in some cases ${ }^{14}$. In recent days, Ivacaftor; a pharmacologic potentiator of CFTR function, had an effect on AWP ${ }^{15}$.

In conclusion most of the AWP patients can be ignored and overlooked in clinical practice if they consult to their doctor solely without water contact test. Therefore, clinicians should evaluate the water contact test as a screening test for patients with suspected AWP, furthermore skin biopsy and CFTR mutation test are recommended. Patients with mild forms of CF may not have symptoms or symptoms develop later, therefore patients identified as having AWP should be screened for CF. If the testing is in the equivocal range (common among heterozygous carriers), screening for CFTR mutations should be considered to help with appropriate genetic counseling.
Herein we report rare cases of AWP in two healthy young women in Korea who evaluated CFTR mutations test but negative and improved without treatment. During follow-up, AWP patients should be periodically checked and observed the possible CF-related clinical symptoms.

\section{CONFLICTS OF INTEREST}

The authors have nothing to disclose.

\section{ORCID}

Hoon Choi, https://orcid.org/0000-0001-8514-3550

In Ho Bae, https://orcid.org/0000-0002-9166-4050

Min Sung Kim, https://orcid.org/0000-0002-8102-6653

Chan Ho Na, https://orcid.org/0000-0001-5259-5382

Bong Seok Shin, https://orcid.org/0000-0001-9618-1763

\section{REFERENCES}

1. Weibel L, Spinas R. Images in clinical medicine. Aquagenic wrinkling of palms in cystic fibrosis. N Engl J Med 2012; 366:e32.

2. Arkin LM, Flory JH, Shin DB, Gelfand JM, Treat JR, Allen J, et al. High prevalence of aquagenic wrinkling of the palms in patients with cystic fibrosis and association with measurable increases in transepidermal water loss. Pediatr Dermatol 2012;29:560-566.

3. Kabashima K, Shimauchi T, Kobayashi M, Fukamachi S, Kawakami C, Ogata $M$, et al. Aberrant aquaporin 5 expression in the sweat gland in aquagenic wrinkling of the palms. J Am Acad Dermatol 2008;59(2 Suppl 1):S28-S32.

4. Kim DW, Woo SH, Kim Jl, Oh YJ, Yun SK, Kim HU, et al. Idiopathic aquagenic wrinkling of the palms in Korean patients. Ann Dermatol 2015;27:776-777.

5. Gild R, Clay CD, Morey S. Aquagenic wrinkling of the palms in cystic fibrosis and the cystic fibrosis carrier state: a case-control study. Br J Dermatol 2010;163:1082-1084.

6. Yoon TY, Kim KR, Lee JY, Kim MK. Aquagenic syringeal acrokeratoderma: unusual prominence on the dorsal aspect of fingers? Br J Dermatol 2008;159:486-488.

7. Lim KS, Ng SK. Aquagenic wrinkling of the palms in a boy with a congenital cardiac anomaly. J Eur Acad Dermatol Venereol 2007;21:985-986.

8. Okuhira H, Matsunaka H, Iwahashi $Y$, Nakamura $Y$, Furukawa F, Yamamoto $Y$. Case report of aquagenic wrinkling of the palms associated with impaired stratum corneum function. J Dermatol 2015;42:913-914.

9. Thomas JM, Durack A, Sterling A, Todd PM, Tomson N. Aquagenic wrinkling of the palms: a diagnostic clue to cystic fibrosis carrier status and non-classic disease. Lancet 2017;389:846.

10. Tolland JP, Boyle J, Hall V, McKenna KE, Elborn JS. Aquagenic wrinkling of the palms in an adult cystic fibrosis 
population. Dermatology 2010;221:326-330.

11. Stewart LC, Doe SJ, Bourke SJ, Leech S. Aquagenic palmar wrinkling as a presenting feature of cystic fibrosis gene dysfunction. Clin Exp Dermatol 2009;34:e647-e649.

12. Khuu PT, Duncan KO, Kwan A, Hoyme HE, Bruckner AL. Unilateral aquagenic wrinkling of the palms associated with aspirin intake. Arch Dermatol 2006;142:1661-1662.

13. Ludgate MW, Patel D, Lamb S. Tobramycin-induced aquagenic wrinkling of the palms in a patient with cystic fibrosis.
Clin Exp Dermatol 2009;34:e75-e77.

14. Adişen E, Karaca F, Gürer MA. Transient reactive papulotranslucent acrokeratoderma in a 50-year-old woman: case report and review of the literature. Am J Clin Dermatol 2008;9:404-409.

15. Ramsey BW, Davies J, McElvaney NG, Tullis E, Bell SC, Dřevínek $\mathrm{P}$, et al.; VX08-770-102 Study Group. A CFTR potentiator in patients with cystic fibrosis and the G551D mutation. N Engl J Med 2011;365:1663-1672. 